\title{
Interrupting the cascade: Orienting contributes to decision making even in the absence of visual stimulation
}

\author{
Claudiu Simion and Shinsuke Shimojo \\ California Institute of Technology, Pasadena, California
}

\begin{abstract}
Most systematic studies of human decision making approach the subject from a cost analysis point of view and assume that people make the highest utility choice. Very few articles investigate subjective decision making, such as that involving preference, although such decisions are very important for our daily functioning. We have argued (Shimojo, Simion, Shimojo, \& Scheier, 2003) that an orienting bias effectively leads to the preference decision by means of a positive feedback loop involving mere exposure and preferential looking. The illustration of this process is a continually increasing gaze bias toward the eventual choice, which we call the gaze cascade effect. In the present study, we interrupt the natural process of preference selection, but we show that gaze behavior does not change even when the stimuli are removed from observers' visual field. This demonstrates that once started, the involvement of orienting in decision making cannot be stopped and that orienting acts independently of the presence of visual stimuli. We also show that the cascade effect is intrinsically linked to the decision itself and is not triggered simply by a tendency to look at preferred targets.
\end{abstract}

Our previous work linked orienting and preference decision making by revealing a particular gaze behavior when observers chose the stimulus they liked in a two-alternative forced-choice task (Shimojo, Simion, Shimojo, \& Scheier, 2003). Namely, whenever observers were asked to make a preference decision as naturally as possible, a continually increasing gaze bias toward the eventual choice was observed. This effect was not present in the case of controlled nonpreference tasks, and its size increased with task difficulty - as if the brain needed a stronger gaze signal to make a more difficult decision. We accounted for the behavior by postulating a positive feedback loop between the structures responsible for decision making on the one hand and the areas controlling orienting on the other. If we imagine that the more we look at a stimulus, the more we like it, according to the well-known process of mere exposure (Kunst-Wilson \& Zajonc, 1980; Moreland \& Zajonc, 1977, 1982; Zajonc, 1968), and that we look longer at stimuli we like (Birch, Shimojo, \& Held, 1985; Fantz, 1964), we realize that the formation of preference could be strongly modulated by gaze.

It thus seems that orienting assists cognition in making preference decisions, with the feedback loop described earlier driving the decision signal above a possible decision threshold (Shimojo et al., 2003). Shimojo et al.'s study eliminated alternative causes for this behavior and provided strong evidence in favor of the proposed model. However, orienting is regarded as a novelty detector and is usually linked to the presence of salient features in the relevant stimuli. Using a gaze-contingent window (peephole) paradigm, we have already shown that holistic stimulus processing is not necessary for the cascade effect to happen (Simion \& Shimojo, 2006). In the Simion and Shimojo study, observers could inspect the stimuli only through a small, gaze-contingent window; they then had to choose the stimulus they liked. Therefore, they had to actively use their gaze to gather information about the stimuli. Naturally, the rate at which observers accumulated visual information was much slower, and the cascade effect was much longer $(7 \mathrm{sec}$ ) than in the 2003 experiments. This result supported the claim that the brain integrates gaze information over time and makes a decision only after a threshold has been passed. Additionally, this result showed that orienting could be entirely memory (and intention) driven, independent of stimulus perception.

Our main claim is that orienting has been incorporated into the mechanism of preference decisions, and its biases contribute to decision making; this claim is similar to what Damasio proposed in the well-known somatic marker hypothesis (Bechara, Damasio \& Damasio, 2000; Damasio, 1996). An interesting question, therefore, is whether the process of decision making, once started, can be disrupted by interfering with the gaze behavior with which it is intrinsically connected. Additionally, none of our previous experiments distinguished preference formation from the actual state of liking a particular stimulus; this state could have been developing unconsciously before the actual choice was made and thus could have driven the cascade effect. The present study was designed to address both of these issues. 
Specifically, observers were asked to choose the more attractive face from a pair. The stimulus presentation time was set by a computerized random-number generator. In some trials, therefore, the observers were able to make a decision before the stimulus was removed from their visual field, whereas in others, the observers had to make the choice after the stimulus had disappeared and they were left gazing at a blank screen. The former situation enabled investigation of gaze behavior after a decision had been made but while the stimuli were still on the presentation screen, thus separating the pre- and postdecision states. If the general state of liking a particular stimulus (e.g., a human face) were triggering or maintaining the cascade effect, we should have observed an increasing or stable gaze bias even after the decision had been made, especially since observers were then conscious of their preference. In contrast, if the cascade effect were maximized at the decision point, after which the gaze bias decreased, then we would link the effect to the process of decision.

The trials in which the faces disappeared from the screen before the observers could choose allowed us to investigate the interruption of the natural course of the gaze bias. Obviously, a decision still had to be made after the stimuli were no longer present, so the orienting behavior in those trials would tell us to what extent the cascade could be disrupted. Studying this disruption was especially important, since after stimulus offset there was no visual target to orient to anymore; orienting was thus decoupled from its original purposes of novelty detection and information gathering, as described by Sokolov and others (Porges, 1995; Posner, Pothbart, \& Digirolamo, 1999; Sokolov, 1963, 1990). One possibility is that the decision could be based on the gaze bias that developed before the end of visual stimulation; in this case, we would see a cascade, albeit of smaller magnitude, locked to the display-off moment. Another possibility is that the mechanism, once started, had to be completed, so we would observe a cascade effect even after stimulus offset. This latter prediction would provide direct evidence for our earlier claim that orienting detaches from its initial purpose of attending to salient features of relevant stimuli and is incorporated into the mechanism of decision making to the point that the orienting process has to be completed before a decision can be made.

\section{METHOD}

Six naive observers participated in this experiment after their written consent was obtained; they were paid $\$ 5$ for their participation. All were students (undergraduate or graduate) at the California Institute of Technology; their mean age was 26.8 years. Each observer was presented with a unique set of $n=40$ pairs of computergenerated human faces; there were thus a total of 240 face pairs, with 20 female and 20 male pairs in each set. Only faces of the same gender were paired. The face generation software was provided by Facegen (Singular Inversions, Vancouver, BC). Prior to the eye-tracking experiment, observers rated each face in the presentation set on a scale from 1 (very unattractive) to 7 (very attractive). A MATLAB script performed the pairing of the faces by adjoining only faces with similar, if not identical, ratings. To limit the effect of familiar- ity, faces were presented very briefly $(500 \mathrm{msec})$ in this preliminary test. In the eye-tracking phase, observers' eye movements were tracked with Eyelink 2 (SR Research Ltd., Osgoode, ON) at $500 \mathrm{~Hz}$ sampling rate, pupil-tracking mode. Face pairs were presented on a CRT computer screen for a random amount of time, according to a MATLAB script that drew the time values from a normal distribution with a mean of $2,700 \mathrm{msec}$ and a standard deviation of $1.0 \mathrm{sec}$. The minimum and maximum presentation times were $800 \mathrm{msec}$ and $5,000 \mathrm{msec}$, respectively. The actual mean presentation time for the 240 face pairs (across all observers) was 3,105 msec.

Observers were asked to choose the more attractive face in each pair. They were informed that the presentation time would be random but were instructed to make a natural, unrushed decision if possible. If the faces were still on the screen at decision time (as in 128 out of 240 trials, called early decision), observers pressed one of two buttons to indicate their choice (face on the left or face on the right). After stimulus offset, they confirmed their decision by pressing the button again. Observers were instructed that they were allowed to change their minds, but in only 1 out of 240 trials did this actually happen. If the faces were taken off the screen before the observers could make a decision (as in 112 out of 240 trials, dubbed late decision), they needed to make a decision (by pressing one of the two choice buttons) while the screen stayed blank. The trials were terminated by the final buttonpress (decision confirmation or decision, respectively).

Faces on the screen were presented side by side, one on the left and one on the right. They were presented as jpeg images spanning $400 \times 400$ pixels, or approximately $17 \times 17 \mathrm{deg} /$ visual angle each.

The gaze likelihood analysis developed elsewhere (Shimojo et al., 2003) was used. We assigned to each time sample point a value of 1 if the gaze was on the chosen face, a 0 if the gaze was on the unchosen face, or a NaN (not a number) otherwise. The likelihood that the chosen face was inspected at time $t$ was thus obtained by averaging across all trials $(N=240)$ and observers. The analysis was performed by aligning trials at various relevant events, such as decision, final buttonpress, or the moment of stimulus offset. Because trial duration varied greatly, samples close to the reference events were averaged across a larger number of trials than were samples farther away from them. This is why each analysis (see Figures 1-3) included a limited number of time sample points, with the number of points arbitrarily chosen to allow averaging across two thirds of the total trials of that experimental condition. NaN values were assigned to samples belonging to saccades and fixations outside of the two faces. Saccades accounted for about $13 \%$ of all samples, and fixations outside the two stimulus faces accounted for another 3\%. Eliminating the $\mathrm{NaN}$ values from the analysis did not change the general profile of the likelihood curves. The significance threshold method described in Simion and Shimojo (2006) was used to identify the start point of a cascade effect. In essence, the significance threshold at each time sample point is the minimum percentage of tosses that would render a coin toss unfair, given a number of tosses equal to the number of trials used to make a likelihood average at that sample point. A gaze cascade effect was defined as the portion of the curve that passed the significance threshold and never returned below it while keeping a monotonically increasing profile.

To analyze the gaze behavior after stimulus offset, only gaze data occurring during the blank screen period were included in the likelihood calculation. The interest areas were defined as the areas previously occupied by the faces, and the same algorithm described above was used. To ensure that any effects were not due to the behavior trailing off from the period when the stimuli were still on the screen, we analyzed only those trials that had at least $300 \mathrm{msec}$ of blank time. All likelihood curves were fitted with four-parameter sigmoid functions, with baseline, slope, elevation, and sigmoid midpoint as the relevant parameters.

\section{RESULTS}

As mentioned in the Method section, the stimulus presentation time in each trial was randomly drawn from a 


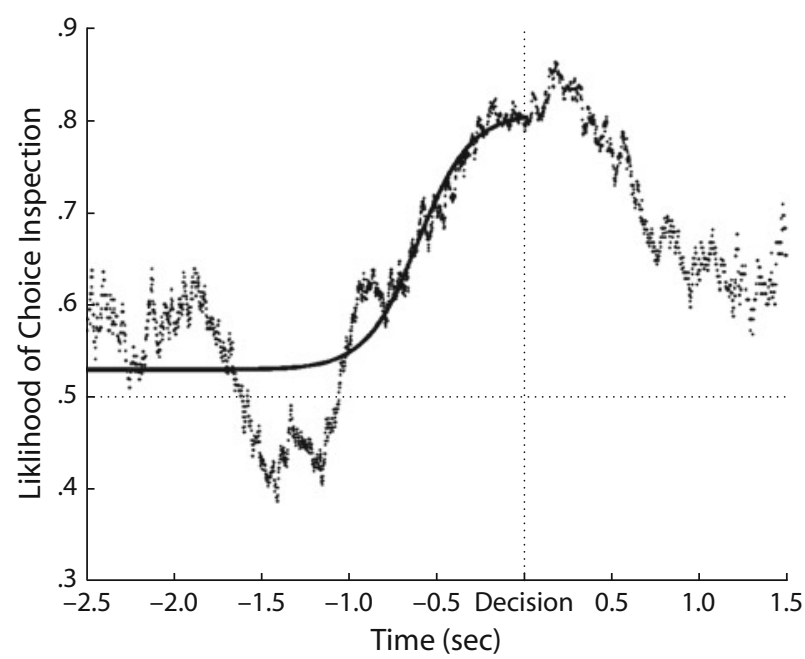

Figure 1. Gaze likelihood curve locked at the decision moment in early decision trials. The solid line represents a four-parameter sigmoid fit. The horizontal line corresponds to the unbiased inspection level $(50 \%)$. The vertical line corresponds to the decision moment, to which all trials were aligned. $R^{2}=.75$. Note that the likelihood goes down after the decision.

normal distribution. The actual mean presentation time was $3,101 \mathrm{msec}(S D=1,205 \mathrm{msec})$. Obviously, the trials in which observers had enough time to decide (early decision trials) were clustered toward the longer presentation times, with a mean of $3,545 \mathrm{msec}(S D=1,028 \mathrm{msec})$. The mean decision time in these trials was $2,395 \mathrm{msec}$ $(S D=846 \mathrm{msec})$. In the early decision trials, observers had to confirm their decisions after the display was off by pressing their choice buttons again. The mean blank time (i.e., between the offset of the stimuli and the buttonpress) was $1,125 \mathrm{msec}(S D=584 \mathrm{msec})$.

The trials that were too short for a decision to be made while the faces were on the screen were called late decision trials. Presentation time in these trials was shorter, with a mean of 2,621 $\mathrm{msec}(S D=1,177 \mathrm{msec})$. The difference in presentation times between early decision and late decision trials was significant $(p<.001)$, as expected. The mean decision time was $3,455 \mathrm{msec}(S D=1,230 \mathrm{msec})$, again significantly higher than the mean for early decision trials $(p<.0001)$. Due to the nature of our division of trials into the two categories, we consider these numbers to have little relevance to the overall conclusions of this study. The mean blank time in this case was $830 \mathrm{msec}$ $(S D=609 \mathrm{msec})$, significantly shorter than the mean in the early decision trials $(p=.0002)$. This comparison is important because the blank screen period underlies functionally distinct phenomena, namely decision confirmation in the early decision trials and the decision itself in the late decision trials. The only common feature between the two types of trials was the motor action (buttonpress) that ended the trial. It might be surprising that confirming a decision took longer than making a decision after stimulus offset, but we have to point out that in the late decision trials, unlike in the early decision trials, the observers were still under pressure to make their choice.
To investigate the orienting behavior in this task, we generated gaze likelihood curves using the gaze analysis method described in a previous study (Shimojo et al., 2003). The gaze likelihood curve for the early decision trials, aligned at the moment of decision, is plotted against time in Figure 1. The time axis extends from $2.5 \mathrm{sec}$ before to $1.0 \mathrm{sec}$ after decision, because the gaze behavior after the choice had been made but before the faces had disappeared from the screen was of particular interest for the purpose of the present study. This postdecision period lasted a mean of 1,184 msec across all trials $(S D=784 \mathrm{msec})$. The presence of a normal cascade effect starting $1 \mathrm{sec}$ before the decision would be consistent with our previous claim that the gaze bias is a prerequisite for decision making. Moreover, the postdecision behavior eliminates the possibility that the bias is due to the general state of liking a particular stimulus. As can be seen, the bias decreases significantly after decision, when subjects are aware of their preference. We conclude, therefore, that the gaze cascade is not a consequence but rather a part of the process that leads to preference decisions.

Since orienting is classically linked to the presence of visual stimulation, we examined the gaze behavior locked to the moment of stimulus offset (the display-off moment). Figure 2 shows the likelihood curve as a function of time before and after the stimulus offset, for both early and late decision trials. In the case of the former, we notice a bias toward choice before the reference point, which was expected since the actual decision took place prior to it. It is important to note that a classic gaze cascade effect is not observed, but this is a direct consequence of aligning the data at stimulus offset rather than at decision. Both likelihood curves are much noisier after the stimulus offset, due to the reduced number of data points to average across (i.e., increased number of NaNs; see Method section).

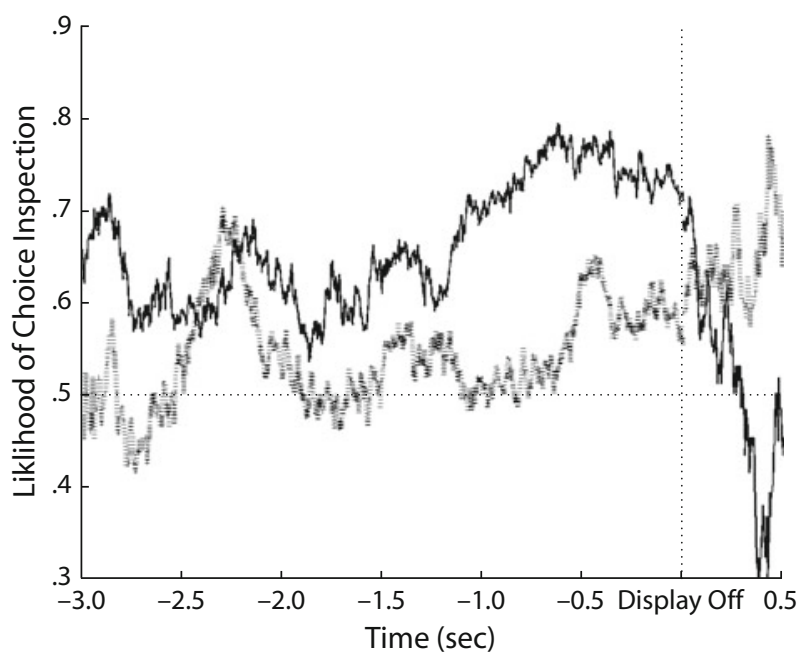

Figure 2. Gaze behavior around stimulus offset. The solid line represents early decision trials; the dashed line, late decision trials. The vertical line represents the reference point (display off). The horizontal line corresponds to the level of unbiased inspection $(50 \%)$. 


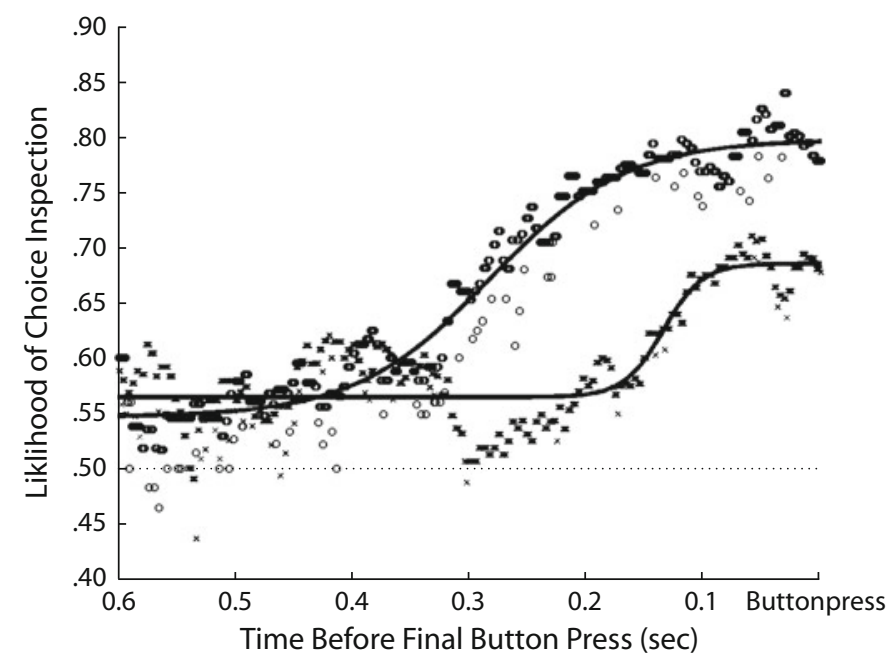

Figure 3. Gaze behavior after stimulus offset, locked at the moment of final buttonpress. Late decision (o) and early decision $(x)$ trials are shown. Solid lines represent four-parameter sigmoid fits. $R^{2}=.95$ for late decision trials and $R^{2}=.73$ for early decision trials. The curves represent behavior occurring exclusively after the stimulus offset.

In the early decision trials, the likelihood curve does not display a consistent gaze bias after stimulus offset. The case of the late decision trials, however, is more interesting. There is no clear gaze cascade before the moment of stimulus offset, but we notice a gaze bias (58\%) toward the eventual choice, so we have reason to believe that we interrupted the gaze cascade effect. There is also a hint of a continuation of the bias after the reference point, which prompts further analysis of the gaze behavior during the poststimulus period in the late decision trials.

The likelihood curves after stimulus offset are presented in Figure 3, plotted against time to the final buttonpress for both early and late decision trials. The reader should note that in this analysis, the gaze data after stimulus offset were aligned at the moment of the final buttonpress, not at the point of stimulus offset, as illustrated in Figure 2. This is why the curves look entirely different. Only the trials in which at least $300 \mathrm{msec}$ separated stimulus offset from buttonpress are included in the analysis, to avoid any trailing off of the gaze behavior from the epoch during which the stimuli were still on the screen.

We notice a shorter (approximately $400 \mathrm{msec}$ ) but clear cascade effect in the late decision trials, as expected in light of our model and since observers had to make a decision. In contrast, results from the early decision trials display a shorter and smaller gaze bias with a plateau-like shape, suggesting internal consistency between the motor action and orienting, a reappearing result from our past studies (Shimojo et al., 2003; Simion \& Shimojo, 2006). The important point is that the curves are significantly different, in terms of both profile and magnitude. The Kolmogorov-Smirnov test confirmed the difference $(d=0.43, p<.001)$. The result again provides strong evidence that orienting is embedded in the mechanism of preference decision making and shows that once started, this contribution process has to be completed before a choice can be made.

\section{DISCUSSION}

Before we begin this section, we will comment on a question that most likely will arise in our readers' minds: How do we know that the final buttonpress (choice) coincides with the actual decision, which may be internal? As a reply, we point out that we view decision making as the entire process, from the moment of stimulus onset to the moment of buttonpress, given the experiment's instructions. Whether an internal decision is made at some point in a trial requires an empirical observation and is difficult to pinpoint. In fact, every saccade that we make can be considered a decision, but this is not the subject of our investigation. Our observers were instructed to press the button as soon as they made their decision, so we consider choice to be the only reliable indicator of decision making in the present study.

Here we investigated, with a single experimental setup, a few aspects of the contribution of orienting to preference decision making; the results significantly expand and strengthen the implications of our previous conclusions. The gaze behavior in the early decision trials (Figure 1) first confirmed our earlier findings, showing that whenever a decision of preference is involved, a cascade effect precedes the choice by approximately $1 \mathrm{sec}$. Second, the fact that the likelihood of inspecting the chosen face decreases after the decision moment argues against a simple, one-directional view on the gaze bias - namely, that it is generated by preferential looking alone (i.e., we tend to look more at what we like) or by the state of liking a particular stimulus itself. We note here that, in light of this result, our positive feedback loop account needs a slight modification, which does not alter our general conclusions. A model that holds that the more we look at a stimulus, the more we like it, and the more we like a stimulus, the more we look at it (Shimojo et al., 2003) 
suggests a cascade effect continuing indefinitely, even after the decision, which is a result that we did not obtain. However, the only adjustment that the model requires is to posit that the positive feedback loop acts only on the still unconscious decision signal, bringing the signal closer to the moment of overt choice.

The likelihood curve locked at the stimulus offset point (display-off moment, Figure 2) in the late decision trials confirmed that our experimental setup was successful in interrupting what we think is the natural process of preference formation, which involves a gaze cascade effect. The dashed curve in Figure 2 shows a significant bias toward the eventually chosen stimulus, oscillating around the $58 \%$ level. However, the decision signal is probably not strong enough yet for the choice to be made and this is why the observers had to complete it after that point.

The most important result of the present study, however, comes from the analysis of the orienting behavior after stimulus offset (Figure 3). As surprising as it sounds, observers completed the gaze cascade by fixating in the locations previously occupied by the faces for at least $830 \mathrm{msec}$, on average. We emphasize here the irrelevance of stimulus presence to the cascade effect itself, as long as a preference decision needs to be made. In a previous study (Simion \& Shimojo, 2006), we showed that mental feature integration, without holistic perception, is enough for the gaze cascade to happen - again, as long as a preference choice was involved. Orienting and the presence of salient stimulus features were only weakly coupled in that experiment, since gaze had to be guided by the observers' memory and by compliance with the task's purpose. Among other results, that experiment seemed to indicate that orienting became so embedded in the process of decision making that stimulus presence was no longer necessary for triggering it, contrary to what Sokolov (1963) described. In our view, the result from the period after stimulus offset in the present study is evidence that the decoupling between orienting and the stimuli it is supposed to detect can be complete. In a flowery description, we say that orienting has been hijacked by the preference decision mechanisms and was used to assist the brain in reaching the overt choice. The gaze bias effectively induces a preference bias.

The difference between the gaze likelihood curves in the early and late decision trials in Figure 3 is also informative. First, this difference shows that a decision is the necessary endpoint of a gaze cascade effect. Second, it argues against the possibility that the cascade effect is just a confirmation of a previously made decision, a point of criticism for our earlier results. If this were the case, the two curves should be similar, since the situation in the early decision trials (represented by crosses in Figure 3 ) is precisely a decision confirmation. The smaller bias toward choice (200 msec before buttonpress) in these trials is also consistent with our earlier findings, which indicated that any selection was generally preceded by a gaze bias, probably for internal, cognitive-motor consistency. The distinction between a selection bias and a gaze cascade effect is a reappearing theme in our studies, but all the evidence indicates that gaze behavior in subjective, emotionally charged decisions goes beyond simple selection bias due to the positive feedback loop between mere exposure and preferential looking.

Taken together, the results of this study nicely complete our story, providing definitive evidence that orienting has been incorporated into the process of preference formation. Our research also suggests that by manipulating active orienting even after visual stimulation has been interrupted, we can influence observers' preferences, an idea with endless potential in advertising, social psychology, and human communication sciences.

\section{AUTHOR NOTE}

Correspondence concerning this article should be addressed to C. Simion, 1451 Beach Park Blvd., \#207, Foster City, CA 94404 (e-mail: claudelu@gmail.com).

\section{REFERENCES}

Bechara, A. H., Damasio, H., \& Damasio, A. R. (2000). Emotion, decision making and the orbitofrontal cortex. Cerebral Cortex, 10, 295-307.

Birch, E. E., Shimojo, S., \& Held, R. (1985). Preferential-looking assessment of fusion and stereopsis in infants aged 1-6 months. Investigative Ophthalmology \& Visual Science, 26, 366-370.

Damasio, A. R. (1996). The somatic marker hypothesis and the possible functions of the prefrontal cortex. Philosophical Transactions of the Royal Society of London: Series B, 351, 1413-1420.

FANTZ, R. L. (1964). Visual experience in infants: Decreased attention to familiar patterns relative to novel ones. Science, 146, 668-670.

Kunst-WiLson, W. R., \& ZAJONC, R. B. (1980). Affective discrimination of stimuli that cannot be recognized. Science, 207, 557-558.

Moreland, R. L, \& Zajonc, R. B. (1977). Is stimulus recognition a necessary condition for the occurrence of exposure effects? Journal of Personality \& Social Psychology, 35, 191-199.

Moreland, R. L., \& ZaJonC, R. B. (1982). Exposure effects in person perception: Familiarity, similarity, and attraction. Journal of Experimental Social Psychology, 18, 395-415.

Porges, S. W. (1995). Orienting in a defensive world: Mammalian modifications of our evolutionary heritage. A polyvagal theory. Psychophysiology, 32, 301-318.

Posner, M. I., Pothbart, M. K., \& Digirolamo, G. J. (1999). Development of brain networks for orienting to novelty. Zh. Vyssh. Nerv. Deiat. Im. I. P. Pavlova, 49, 715-22.

Shimojo, S., Simion, C., Shimojo, E., \& Scheier, C. (2003). Gaze bias both reflects and influences preference. Nature Neuroscience, 6, 1317-1322.

Simion, C., \& Shimojo, S. (2006). Early interactions between orienting, visual sampling and decision making in facial preference. Vision Research, 46, 3331-3335.

SoKoLov, E. N. (1963). Higher nervous functions: The orienting reflex. Annual Review of Physiology, 25, 545-580.

SoKolov, E. N. (1990). The orienting response, and future directions of its development. Pavlovian Journal of Biological Science, 25, 142-150.

ZaJonc, R. B. (1968). Attitudinal effects of mere exposure. Journal of Personality \& Social Psychology, 9(2), 1-27.

(Manuscript received January 31, 2006; revision accepted for publication October 9, 2006.) 